Artículo

\title{
Residuos de repollo para biocontrol de Fusarium spp. en el cultivo de tomate
}

\author{
Berta F. Civieta-Bermejo ${ }^{1}$ \\ Marcelino Cabrera-De la Fuente ${ }^{2}$ \\ Susana González-Morales ${ }^{3}$ \\ Adalberto-Benavides Mendoza ${ }^{2}$ \\ Alberto Sandoval-Rangel ${ }^{2 \S}$ \\ ${ }^{1}$ Doctorado en Ciencias en Agricultura Protegida. ${ }^{2}$ Departamento de Horticultura-Universidad Autónoma \\ Agraria Antonio Narro. Calzada Narro núm. 1923, Saltillo, Coahuila, México. CP. 25315. Tel. 8444110303. \\ ${ }^{3}$ Catedrático CONACYT. \\ ${ }^{\S}$ Autor para correspondencia: alberto.sandoval@uaaan.edu.mx.
}

\section{Resumen}

Uno de los principales problemas fitosanitarios en la producción de tomate o jitomate (Solanum lycopersicon Mill.) donde se utiliza el suelo como medio de cultivo es Fusarium spp., el control químico es el método frecuentemente utilizado para su manejo; con impactos económicos y ambientales importantes. Los objetivos del presente estudio fueron: evaluar el repollo como cultivo de rotación y la incorporación de los residuos al suelo sobre la incidencia de la marchitez permanente en plantas de tomate e incidencia de Fusarium spp., en el suelo y evaluar la supresión de Fusarium spp., mediante la aplicación de subproductos de hojas y tallos de repollo. El trabajo se realizó en dos etapas: en la primera se documentó la evolución de Fusarium spp., en un cultivo comercial de tomate, establecido bajo condiciones de malla antiáfidos y repollo como cultivo de rotación, durante el periodo de 2012 a 2019. En la segunda etapa, se evaluó un extracto acuoso y un polvo deshidratado, obtenidos de hojas y tallos residuales de repollo. Los resultados muestran que el repollo utilizado como cultivo de rotación y la incorporación al suelo de las hojas y tallos residuales, redujeron la incidencia de marchitez permanente en el cultivo de tomate y suprimieron eficazmente Fusarium spp. Asimismo, el polvo deshidratado obtenido de estos residuos mostró un efecto similar sobre el control de Fusarium spp., al obtenido en los tratamientos con metilditiocarbamato de sodio.

Palabras clave: biofumigación, fusariosis, incidencia.

Recibido: abril de 2021

Aceptado: julio de 2021 


\section{Introducción}

En la producción de tomate o jitomate (Solanum lycopersicon Mill.), utilizando suelo como medio de cultivo, uno de los principales problemas fitosanitarios es la marchitez vascular o fusariosis causada por hongos del género Fusarium spp., (Ma et al., 2013). La incidencia y severidad de la enfermedad se incrementa cuando el cultivo se desarrolla en invernadero o mallas, debido al monocultivo y la intensidad de la explotación. Para el control de esta enfermedad, lo más común es la aplicación al suelo de fumigantes y fungicidas químicos, como es el metil ditiocarbamato de sodio, comercialmente llamado: Metam, Busan, Fumisol, Nemasol, Raisan.

Estos productos han permitido mantener la producción de este cultivo, sin embargo, provocan un importante impacto tanto económico como ambiental. El impacto económico oscila entre los \$7 000.00 a $\$ 40000.00$ pesos por hectárea, dependiendo de la cantidad aplicada, normalmente las recomendaciones inician con $125 \mathrm{~L} \mathrm{ha}^{-1}$ y van aumentando hasta llegar a los $800 \mathrm{~L} \mathrm{ha}^{-1}$, además del incremento de fungicidas y acciones adicionales, como tapetes sanitarios y eliminación de plantas enfermas para reducir la diseminación del patógeno, una vez que está establecido el cultivo (Carmona y Sautua, 2017). El impacto ambiental se debe a una disminución importante de la microbiota nativa del suelo y el posterior incremento de las poblaciones de patógenos, que comienzan con daños a algunas plantas y posteriormente se expanden, hasta cubrir regiones completas (Abdel-Monaim et al., 2011).

Por otro lado, cada vez es mayor la demanda de alimentos inocuos producidos sin el uso de agroquímicos o con el menor uso de ellos (Gottschalk y Leistner, 2012; Bryła, 2016). Todo esto ha llevado a buscar alternativas para el manejo de enfermedades mediante un control más compatible con el medio ambiente, más inocuos y viables (Ram et al., 2018; Zavaleta-Mejía, 1998).

Una alternativa al control químico de esta enfermedad, podría ser el uso de sustancias naturales con propiedades antagonistas a patógenos especialmente del suelo. Las crucíferas son un claro ejemplo de esto, debido a que poseen propiedades antimicrobianas, relacionada con el alto contenido de compuestos azufrados en sus tejidos denominados glucosinolatos (Brown y Morra, 2005; Rodríguez et al., 2013), además de la enzima glucohidrolasa tioglucosido, llamada mirosinasa (Morra y Kirkegaard, 2002), esta enzima hidroliza a los glucosinolatos transformándolos en una aglicona inestable, que posteriormente sufre modificaciones para dar lugar a compuestos volátiles tóxicos como sulfidrilos, nitrilos, tiocianatos, isiotiocianatos, entre otros. Cada especie de crucífera tiene diferentes clases y concentraciones de glucosinolatos (Rosa, 1997; Brown y Morra, 2005; Campas-Baypoli et al., 2009; Rodríguez et al., 2013), los cuales se mantienen inclusive en residuos deshidratados (Lazzeri y Dallavalle, 2004).

La eficacia de los residuos de diferentes plantas del género Brassica incorporados al suelo para la supresión de Fusarium spp., ha sido documentada en campo (Gilardi et al., 2016; Prasad y Kumar, 2017; Campanella et al., 2020). El uso de esta alternativa implica usar las plantas de crucíferas, como cultivo de rotación, lo cual se dificulta en el sistema de agricultura protegida, por la poca disponibilidad de tiempo, por ello, se propone evaluar otras opciones, especialmente extractos líquidos y deshidratados obtenidos de hojas y tallos residuales de repollo, como opción al control químico y a quienes no puedan o no deseen utilizar cultivos de rotación para el manejo de Fusarium spp. 
Basado en lo anterior, los objetivos de esta investigación fueron: Evaluar el efecto del uso de repollo como cultivo de rotación y la incorporación de los residuos al suelo, en la incidencia de la marchitez permanente en plantas de tomate y en las poblaciones de Fusarium spp., en el suelo y evaluar la supresión de la marchitez permanente en plantas de tomate e incidencia de Fusarium spp., en el suelo mediante la aplicación de subproductos de hojas y tallos de repollo.

\section{Materiales y métodos}

\section{El estudio se realizó en dos etapas}

Primera etapa: evaluación del repollo como cultivo de rotación y la incorporación de los residuos al suelo sobre la incidencia de la marchitez permanente en plantas de tomate e incidencia de Fusarium spp. en el suelo. El experimento se condujo por siete años (2012-2019), en una plantación comercial de tomate tipo saladette F1 (de crecimiento indeterminado), plantado en suelo desnudo, en una superficie de 6 ha, cubierta con malla antiáfidos, ubicada en el Rancho Poca Luz 1, en San Cristóbal, municipio de Catorce, San Luis Potosí, México. Los ciclos de producción de tomate fueron de abril a septiembre y el repollo (cultivo de rotación) de octubre a febrero. En febrero se cosecho el repollo, las hojas extendidas y tallos se incorporaron al suelo como residuos de cosecha.

A las plantas con marchitez permanente, se les aplicaron fungicidas (tiabendazol, carbendazim, tiofanato metílico) directamente al cuello de la planta, solo para contener la diseminación del patógeno. Previo al inicio de cada ciclo de producción de tomate, se estimó la población de Fusarium en suelo, en un laboratorio certificado (CIFEF, 2018), de 2011 a 2016, se realizó una muestra para la superficie y de 2017 a 2019 se realizaron cuatro muestreos. Se evaluó la incidencia de fusariosis en plantas y se verificó por el CIFEF. De 2012 a 2016 se realizó un registro de plantas con marchitez y muertas en la superficie al final del ciclo de producción, mientras que de 2017 a 2019, se muestrearon plantas con marchitez en la misma superficie, pero dividida en cuatro secciones. Los datos de estos muestreos se usaron para el análisis de incidencia de Fusarium spp.

Segunda etapa: consistió en evaluar la supresión de Fusarium spp., en el suelo mediante la aplicación de subproductos de hojas y tallos de repollo. Se realizó en las instalaciones del departamento de horticultura de la Universidad Autónoma Agraria Antonio Narro (UAAAN), en Saltillo, Coahuila, México. Para la obtención de polvo deshidratado, se colectaron hojas y tallos del cultivo de repollo (70-30 p/p), al terminar la cosecha de repollo, la planta se cortó de la base del tallo, y ambos tejidos se expusieron directamente al sol hasta registrar un peso constante (aproximadamente 8 días), luego se molieron en un molino manual y se cribaron usando una malla de $3 \mathrm{~mm}$. El extracto acuoso: se obtuvo de hojas y tallos frescos, los tejidos se licuaron en una licuadora doméstica, por tres min a $23{ }^{\circ} \mathrm{C}$, a razón de $200 \mathrm{~g}$ de hojas y tallos frescos por $100 \mathrm{ml}$ de agua destilada, después se filtró usando papel filtro estándar, con un tamaño de poro de $10 \mu \mathrm{m}$, el filtrado se conservó en un matraz en refrigeración a $4{ }^{\circ} \mathrm{C}$ hasta su uso.

Adicionalmente, en esta prueba se agregó un tratamiento con hojas y tallos segmentados, similar a la obtenida en la plantación comercial. Para lo cual se utilizaron hojas y tallos frescos que fueron troceados aun tamaño de $10 \mathrm{~cm}$, con un cuchillo de acero inoxidable. Los tratamientos evaluados en esta etapa se describen en el Cuadro 1. Cada tratamiento consistió en 24 macetas de polietileno de $10 \mathrm{~L}$ (repeticiones) con suelo infestado y cada maceta contenía una planta. 
Cuadro 1. Tratamientos a base del extracto acuoso y el polvo deshidratado de hojas y tallos residuales de repollo, evaluados en la supresión de Fusarium spp., en plantas de tomate.

\begin{tabular}{cc}
\hline Número & Descripción del tratamiento \\
\hline T1 & Suelo esterilizado (testigo absoluto $)$ \\
T2 & Suelo infestado con Fusarium spp. $\left(1000 \mathrm{ufc}^{-1}\right)(\mathrm{CIFEF}, 2018)$. \\
T3 & Suelo infestado + Metil ditiocarbamato de sodio* $\left(1 \mathrm{~cm}^{3} \mathrm{~L}^{-1}\right.$ de agua $)$ \\
T4 & Suelo infestado + segmentos de repollo fresco $\left(150 \mathrm{~g} \mathrm{maceta}^{-1}\right)$ \\
T5 & Suelo infestado + extracto acuoso de repollo $\left(210 \mathrm{~cm}^{3}\right.$ maceta $\left.^{-1}\right)$ \\
T6 & Suelo infestado + polvo deshidratado de repollo $\left(15 \mathrm{~g} \mathrm{maceta}^{-1}\right)$ \\
\hline
\end{tabular}

* = producto comercial Busan 1020. (Metam sodio al 33\%). Summit Agro, México.

Para la aplicación de los tratamientos con polvo deshidratado y segmentos (trozos) de repollo, previamente se llenaron las macetas con suelo infestado hasta la mitad, luego se adicionaron los tratamientos y se cubrieron con suelo hasta llenarse: para los tratamientos del extracto acuoso y el metil ditiocarbamato de sodio (MDTCNa) se aplicaron en el agua de riego, disolviendo los tratamientos en $2 \mathrm{~L}$ de agua. El MDTCNa, se aplicó 21 días antes del trasplante, para el resto de los tratamientos el trasplante se realizó inmediatamente después de su aplicación. Para la esterilización del suelo se utilizó una autoclave Yamato SQ810C.

En esta etapa se evaluó: incidencia, medida como plantas con síntomas de fusarosis (marchitez permanente y plantas muertas), adicionalmente al final del cultivo se tomó un disco de la base del tallo, este disco se lavó con agua destilada en la parte exterior para evitar contaminación con otros microorganismos, posteriormente se seccionaron en cuatro partes, para exponer la parte interior del tallo, y se puso en un medio de cultivo PDA, a las $72 \mathrm{~h}$ se revisaron los crecimientos, para verificar la presencia de Fusarium spp. Para estimar productividad y calidad del tomate, se contó número y peso de frutos en cada corte y para calidad se determinó el peso promedio de los frutos.

Los datos obtenidos se sometieron a un análisis de varianza (Andeva) ( $p \geq 0.05$ y 0.01 ) bajo un diseño de bloques completos al azar y una prueba de separación de medias Tukey ( $p \geq 0.05$ ), (Zar, 2010), utilizando el software Statistica versión 7.0.

\section{Resultados y discusión}

Primera etapa experimental: evaluación del repollo como cultivo de rotación y la incorporación de los residuos al suelo sobre la incidencia de la marchitez permanente en plantas de tomate e incidencia de Fusarium spp., en el suelo

Población de Fusarium en el suelo. Con base en los análisis de suelo al inicio de cada ciclo de tomate, se identificaron las especies de $F$. oxysporum y $F$. solani (CIFEF, 2018) y de manera general se observó una tendencia a la baja de ambas poblaciones de Fusarium en el suelo, al usar 
repollo como cultivo de rotación y la incorporación de los tallos y hojas residuales al suelo. Asimismo, de manera general, la mayor reducción se observó en los primeros dos ciclos de producción, y a partir del tercer ciclo la población se mantuvo en valores medios de $1000 \mathrm{ufc} \mathrm{kg}^{-1}$ (Figura 1).

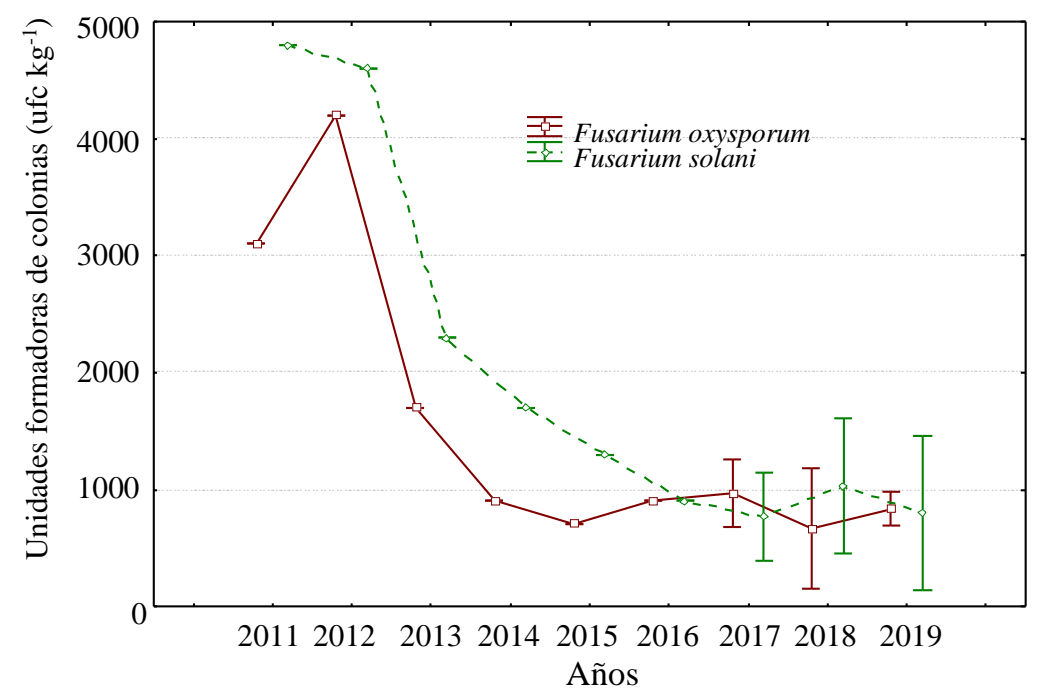

Figura 1. Dinámica poblacional de Fusarium oxysporum y Fusarium solani en el suelo, utilizado para el cultivo de tomate y repollo como cultivo de rotación.

La disminución de las poblaciones de $F$. oxysporum y $F$. solani parece estar relacionada, por un lado, al uso del repollo como cultivo de rotación, lo cual limita la disponibilidad de alimento, aprovechando la especificidad documentada de estas especies de Fusarium hacia las solanáceas y no así para crucíferas (Gil, 1972; Fahey, 2001), siendo la disponibilidad de alimento la principal condición para el crecimiento de las poblaciones de cualquier organismo (Bell et al., 1991).

Por otra parte, las poblaciones de Fusarium pudieron verse afectadas por los productos derivados de la descomposición de las hojas y los tallos de repollo incorporados al suelo como residuos o esquilmos, debido a que los glucosinolatos y la mirosinasa al entrar en contacto derivan en una serie de compuestos como: sulfhidrilos, nitrilos, tiocianatos e isotiocianatos, estos últimos con capacidad biofumigante o biocida contra hongos del suelo incluido Fusarium spp. (Fahey et al., 2001; Morra y Kirkegaard, 2002; Lazzeri et al., 2004; Pérez, 2014).

Incidencia de Fusarium spp. La incidencia inicial de plantas con marchitez permanente o muertas fue de $11.3 \%$. Después de la primera rotación con repollo y la incorporación de los residuos, este valor disminuyó significativamente hasta $6.8 \%$. En los ciclos subsecuentes esta incidencia fue variable, pero con una tendencia a la baja. Durante los ciclos de 2017 a 2019, la incidencia se mantuvo más estable y por debajo cercana al 4\% (Figura 2). 


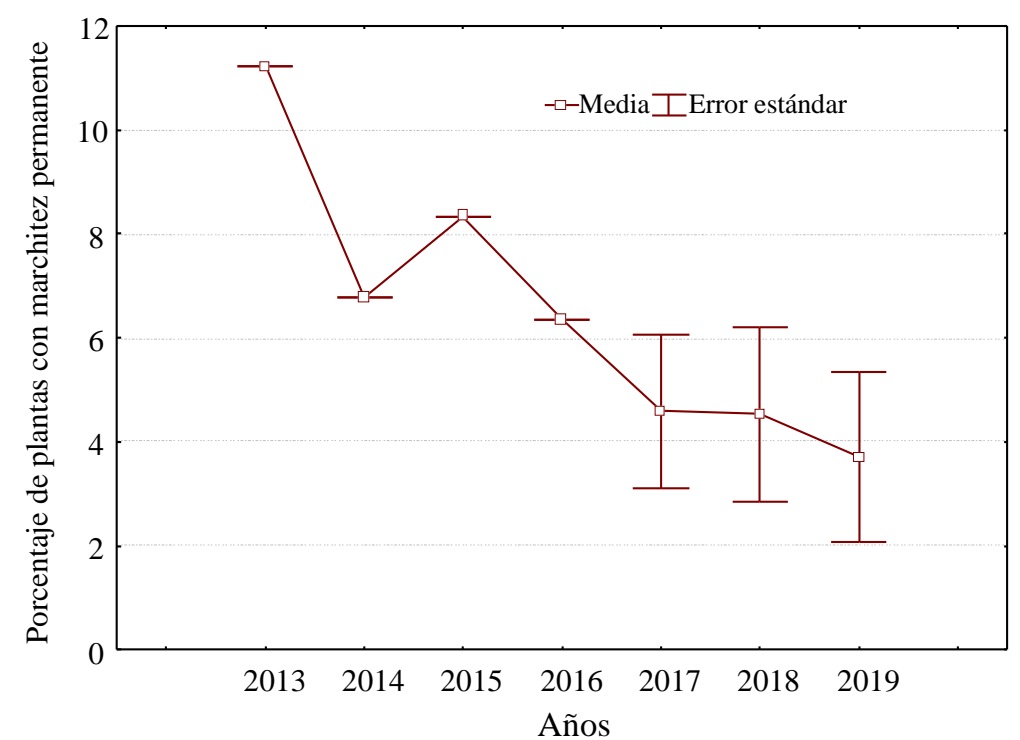

Figura 2. Incidencia de marchitez permanente causada por Fusarium spp., en el cultivo de tomate, utilizando repollo como cultivo de rotación y la incorporación de sus residuos al suelo. Durante un periodo de siete años de muestreo.

La incidencia de marchitez permanente a través del ciclo del cultivo de tomate 2017- 2019 (Figura $3)$, evidenció porcentajes bajos $(<0.3)$ en los primeros meses después de haberse establecido el cultivo de tomate y se incrementó rápidamente en el mes de junio, y se mantuvo hasta el mes de agosto: Lo anterior pudo deberse a las lluvias registradas habitualmente en junio, aunado a que los meses de junio a agosto son los más calurosos del año, en la región donde se desarrolló el experimento, lo cual favorece el desarrollo de Fusarium spp., ya que se ha documentado que este patógeno crece óptimamente en climas cálidos y suelos húmedos (Agrios, 1985). Mientras que la incidencia tiende a bajar en septiembre y octubre, posiblemente al decremento de la temperatura.

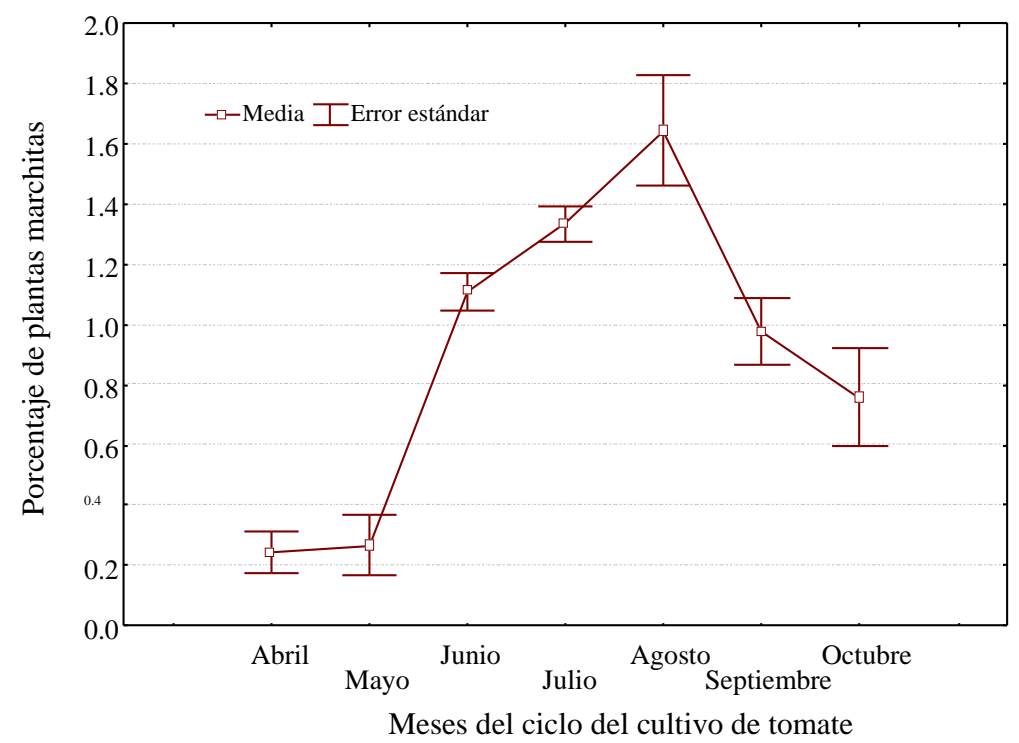

Figura 3. Dinámica de la incidencia de marchitez permanente, en plantas de tomate durante el ciclo del cultivo. Se muestra la media de tres ciclos (2017-2019). 
Por otra parte, el tomate cultivado en las condiciones anteriormente descritas empieza a producir a los 65 días después del trasplante, en esta etapa la planta incrementa la acumulación de carbohidratos y como consecuencia la susceptibilidad de la planta al ataque de hongos fitopatógenos, lo que pudo verse reflejado en el incremento en la incidencia de marchitez permanente por Fusarium a partir de junio, con una tendencia alta hasta agosto, en cada ciclo del cultivo.

\section{Segunda parte: evaluación de un extracto acuoso y un polvo deshidratado, obtenidos de hojas y tallos residuales de repollo}

Las hojas y tallos deshidratados de repollo redujeron significativamente la incidencia de la enfermedad y la población de Fusarium spp., en el suelo (Cuadro 2), atribuido a que tanto los glucosinolatos como la mirosinasa permanecieron en los tejidos deshidratados y que la síntesis de tiocianatos e isotiocianatos inicio al hidratarse con la humedad del suelo (Sarwar et al., 1998; Caballero, 2017). Los valores de incidencia obtenidos con este tratamiento fueron similares a los obtenidos con el fumigante convencional MDTCNa (Cuadro 2). El extracto acuoso, tuvo un efecto limitado respecto al suelo sin tratamiento (T2), posiblemente debido al proceso de licuado, que pudo acelerar el proceso de síntesis de tiocianatos e isotiocianatos, al permitir el contacto rápido de los glucosinolatos con la mirosinasa, además, al tratarse de compuestos volátiles, pudo haber disminuido drásticamente su concentración (Wu et al., 2015).

Cuadro 2. Incidencia de marchitez permanente por Fusarium spp., en plantas de tomate y colonización de Fusarium en suelo tratado con subproductos de hojas y tallos de repollo.

\begin{tabular}{cccc}
\hline Tratamientos & Plantas marchitas $(\%)$ & Plantas muertas $(\%)$ & $\begin{array}{c}\text { Colonización } \\
\text { Suelo ufc } \mathrm{kg}^{-1}\end{array}$ \\
\hline T1 & $4 \mathrm{~d}$ & $0 \mathrm{~d}$ & $33 \mathrm{c}$ \\
T2 & $40 \mathrm{a}$ & $40 \mathrm{a}$ & $2400 \mathrm{a}$ \\
T3 & $4 \mathrm{~d}$ & $4 \mathrm{c}$ & $300 \mathrm{ab}$ \\
T4 & $12 \mathrm{c}$ & $8 \mathrm{c}$ & $566.66 \mathrm{ab}$ \\
T5 & $28 \mathrm{~b}$ & $20 \mathrm{~b}$ & $1833 \mathrm{a}$ \\
T6 & $8.8 \mathrm{~cd}$ & $4 \mathrm{c}$ & $500 \mathrm{ab}$ \\
\hline
\end{tabular}

Letras diferentes en la misma columna indican diferencia estadística en las medias de acuerdo con la prueba de Tukey $(p \geq 0.05)$. ufc $=$ unidades formadoras de colonias.

La baja presencia de plantas con marchitez posiblemente esté relacionada a la sincronía de la síntesis tiocianatos e isotiocianatos y el aumento en la población Fusarium spp., porque la infección no es inmediata, así mismo, para que ocurra la síntesis de isotiocianatos debe darse el proceso de hidratación del polvo del repollo, ya que debe haber un medio acuoso para que la enzima se encuentre con los glucosinolatos.

\section{Efecto sobre el rendimiento y calidad}

El rendimiento y calidad de las plantas de tomate tratadas se muestran en el Cuadro 3. el rendimiento fue diferente, por la eliminación de plantas muertas o bien por la reducción de productividad de las plantas con marchitez. 
El tratamiento con extracto acuoso de repollo fue el menos eficiente, atribuido posiblemente a la volatilización de los tiocianatos e isotiocianatos (Wu et al., 2015; Hashimoto et al., 2020), por lo que la planta se vio afectada mas severamente por la enfermedad y consecuentemente se redujo su productividad (Rodríguez-Araujo et al., 2010).

El tratamiento a base de repollo deshidratado en polvo (T6), mostró el mayor rendimiento, inclusive mayor al obtenido en las plantas tratadas con MDTCNa (Cuadro 3). Esta respuesta puede estar relacionada, además de la reducción de plantas con marchitez, a la adición de materia orgánica, que ocurre al incorporar el polvo deshidratado de las hojas y tallos de repollo. Al adicionar $15 \mathrm{~g}$ por maceta de $10 \mathrm{~L}$ de suelo, equivale a suministrar $4500 \mathrm{~kg} \mathrm{ha}^{-1}$, de materia orgánica seca, lo cual es similar a la cantidad de residuos de cosecha que se incorporan al suelo cuando se utiliza repollo como cultivo de rotación.

El repollo produce de 80 a 100 toneladas de materia verde, de las cuales aproximadamente la mitad son residuos que quedan en el campo, esta cantidad de materia orgánica es suficiente para inducir cambios significativos en las propiedades fisicoquímicas y en la fertilidad de los suelos y por lo tanto en la mejora de los cultivos (Salas-Pérez et al., 2016; Oldfield et al., 2017; Pinedo et al., 2018).

Cuadro 3. Efecto de los subproductos de hojas y tallos de repollo aplicados para la supresión de Fusarium spp., y su impacto en el rendimiento y calidad de tomate.

\begin{tabular}{ccccc}
\hline Tratamientos & Núm. de frutos Peso promedio del fruto $(\mathrm{g})$ Rendimiento (kg) & $\begin{array}{c}\text { Reducción del } \\
\text { rendimiento (\%) }\end{array}$ \\
\hline T1 & $202.34 \mathrm{a}$ & $114.16 \mathrm{ab}$ & $23.09 \mathrm{a}$ & $+5.19 \mathrm{a}$ \\
T2 & $103.5 \mathrm{~b}$ & $101.87 \mathrm{~b}$ & $10.54 \mathrm{a}$ & $-51.85 \mathrm{a}$ \\
T3 & $195.54 \mathrm{ab}$ & $111.95 \mathrm{ab}$ & $21.89 \mathrm{a}$ & $0 \mathrm{a}$ \\
T4 & $175.13 \mathrm{ab}$ & $109.54 \mathrm{~b}$ & $19.18 \mathrm{a}$ & $-12.38 \mathrm{a}$ \\
T5 & $145.79 \mathrm{ab}$ & $108.7 \mathrm{~b}$ & $15.84 \mathrm{a}$ & $-27.63 \mathrm{a}$ \\
T6 & $175.42 \mathrm{ab}$ & $127.54 \mathrm{a}$ & $22.37 \mathrm{a}$ & $+2.19 \mathrm{a}$ \\
\hline
\end{tabular}

Letras diferentes en la misma columna indican diferencia estadística en las medias de acuerdo con la prueba Tukey $(p \geq 0.05)$. ufc $=$ unidades formadoras de colonias.

\section{Conclusiones}

El repollo (Brassica oleracea var. capitata L.), utilizado como cultivo de rotación y la incorporación de sus hojas y tallos residuales al suelo, disminuyó la población de Fusarium oxysporum y Fusarium solani en el suelo y redujo la incidencia de plantas con marchitez permanente causada por Fusarium spp., en tomate (Solanum lycopersicon Mill.) cultivado en suelo y malla antiáfidos. Asimismo, el polvo deshidratado obtenido de las hojas y tallos residuales de repollo disminuyó la incidencia de fusariosis en plantas de tomate y suprimió la población de Fusarium spp., en el suelo, de forma similar al metilditiocarbamato de sodio. 


\section{Literatura citada}

Abdel-Monaim, M. F.; Abo-Elyousr, K. A. M. and Morsy, K. M. 2011. Effectiveness of plant extracts on suppression of damping-off and wilt diseases of lupine (Lupinus termis Forsik). Crop Protec. 30(2):185-191. https://doi.org/10.1016/j.cropro.2010.09.016.

Agrios, N. G. 1985. Fitopatología. Marchitamientos por Fusarium. Editorial Limusa. México, DF. 371-375 pp.

Bell, G. and Koufopanou, V. 1991. The architecture of the life cycle in small organisms. Philosophical Transactions of the Royal Society of London. Series B. Biological Sci. 332(1262):81-89.

Brown, J. y Morra, M. J. 2005. Glucosinolate-containing seed meal as a soil amendment to control plant pests:2000-2002. (No. NREL/SR-510-35254). National Renewable Energy Lab. Golden. CO (US). 199 p.

Bryła, P. 2016. Organic food consumption in Poland: motives and barriers. Appetite. 105:737-746. https://doi.org/10.1016/j.appet.2016.07.012.

Caballero, B. L.; Márquez, C. J. y Betancur, M. I. 2017. Efecto de la liofilización sobre las características caracteristicas físico-químicas del ají rocoto (Capsicum pubescens $\mathrm{R} \& \mathrm{P}$ ) con o sin semilla. Bioagro. 29(3):225-234.

Campanella, V.; Mandal, C.; Angileri, V. and Miceli, C. 2020. Management of common root rot and Fusarium foot rot of wheat using Brassica carinata break crop green manure. Crop Protection. 130:105073. https://doi.org/10.1016/j.cropro.2019.105073.

Campas-Baypoli, O. N.; Bueno-Solano, C.; Martínez-Ibarra, D. M.; Camacho-Gil, F.; Villa-Lerma, A. G.; Rodríguez-Núñez, J. R. y Sánchez-Machado, D. I. 2009. En vegetales crucíferos. Archivos Latinoam. Nutrición. 59(1):95-100.

Carmona, M. y Sautua, F. 2017. La problemática de la resistencia de hongos a fungicidas. causas y efectos en cultivos extensivos. Rev. Facultad de Agronomía UBA. 37(1):1-19.

CIFEF. 2018. Centro Internacional de Servicios Fitosanitarios, SA de CV. Laboratorio integral de diagnóstico fitosanitario. Cedula de aprobación de SAGARPA: 97-705-002 DFG. Saltillo, Coahuila, México.

Gilardi, G.; Pugliese, M.; Gullino, M. L. and Garibaldi, A. 2016. Effect of different organic amendments on lettuce fusarium wilt and on selected soilborne microorganisms. Plant Pathol. 65(5):704-712.

Gottschalk, I. and Leistner, T. 2012. Consumer reactions to the availability of organic food. Inter. J. Consumer Studies. 37(2):136-142. https://doi.org/10.1111/j.1470-6431.2012. 01101.x.

Hashimoto, Y.; Sakamoto, H.; Asai, H.; Yasoshima, M.; Min, H. and Koichi, L. 2020. The effect of fumigation with microencapsulated allyl isothiocyanate in a gas barrier bag against Solenopsis invicta ( Hymenoptera : Formicidae). Appl. Entomol. Zoology. 55(3):345-350. https://doi.org/10.1007/s13355-020-00684-9.

Lazzeri, L.; Curto, G.; Leoni, O. and Dallavalle, E. 2004. Effects of glucosinolates and their enzymatic hydrolysis products via myrosinase on the root-knot nematode Meloidogyne incognita (Kofoid et White) Chitw. J. Agric. Food Chem. 52(22):6703-6707. https://doi.org/10.1021/jf030776u.

Ma, L. J.; Geiser, D. M.; Proctor, R. H.; Rooney, A. P.; O’Donnell, K.; Trail, F. and Kazan, K. 2013. Fusarium pathogenomics. Annual Review Microbiol. 67(1):399-416. https://doi.org/10.1146/annurev-micro-092412-155650. 
Morra, M. J. and Kirkegaard, J. A. 2002. Isothiocyanate release from soil-incorporated Brassica tissues. Soil Biol. Biochem. 34(11):1683-1690. https://doi.org/10.1016/S00380717(02)00153-0.

Oldfield, E. E.; Wood, S. A. and Bradford, M. A. 2017. Direct effects of soil organic matter on productivity mirror those observed with organic amendments. Plant and Soil. 423(1):363-373.

Pinedo, M.; Abanto-Rodríguez, C.; Oroche, D.; Paredes, E.; Bardales-Lozano, R. M.; Alves, E. y Vargas, J. 2018. Mejoramiento de las característias agronómicas y rendimiento de fruto de camu-camu con el uso de biofertilizantes en Loreto, Perú. Scientia Agropecuaria, 9(4):527-533.

Prasad, P. and Kumar, J. 2017. Management of fusarium wilt of chickpea using brassicas as biofumigants. Legume Res. 40(1):178-182. https://doi.org/10.18805/lr.v0i0.7022.

Ram, R. M.; Keswani, C.; Bisen, K.; Tripathi, R.; Surya, P. and Singh, H. B. 2018. Biocontrol technology: eco-friendly pproaches for sustainable agriculture. omics technologies and bioengineering. Academic Press. 177-190 pp. https://doi.org/10.1016/B978-0-12-8158708.00010-3.

Rodríguez-Araujo, E. A.; Bolaños-Benavides, M. M. and Menjivar-Flores, J. C. 2010. Effect of the fertilization on the nutrition and yield of the red pepper (Capsicum spp.) in the Valley of the Cauca, Colombia. Acta Agronómica. 59(1):55-64. http://www.redalyc.org/pdf/ 1699/169916223005.pdf.

Rodríguez, M. K. A.; Monreal,V. C. T.; Huerta, D. J.; Soria, C. J. C. y Jarquín, G. R. 2013. Aporte de microorganismos benéficos por la incorporación al suelo de residuos deshidratados de col (Brassica oleracea var capitata) y su Efecto en el pH. Rev. Mex. Fitopatol. 31(1):29-44.

Rosa, E. A. S. 1997. Daily variation in glucosinolate concentrations in the leaves and roots of cabbage seedlings in two constant temperature regimes. J. Sci. Food Agric. 73(3):364-368. https://doi.org/10.1002/(SICI)1097-0010(199703)73:3<364::AID-JSFA742>3.0.CO;2-O.

Salas-Pérez, L.; González, J. A.; Garcia, M.; Sifuentes, I. E.; Parra, T. S. y Preciado, R. P. 2016. Calidad biofísica y nutracéutica de frutos de tomate producido con sustratos orgánicos. Nova Sci. 8(17):310-325.

Sarwar, M.; Kirkegaard, J. A.; Wong, P. T. W. amd Desmarchelier, J. M. 1998. Biofumigation potential of brassicas III. In vitro toxicity of isothiocyanates to soil-borne fungal pathogens. Plant and Soil. 201(1):103-112. https://doi.org/10.1023/A:1004381129991.

Wu, H.; Xue, N.; Hou, C.; Feng, J. and Zhang, X. 2015. Microcapsule preparation of allyl isothiocyanate and its application on mature green tomato preservation. Food Chem. 175:344-349. https://doi.org/10.1016/j.foodchem.2014.11.149.

Zar, J. H. 2010. Biostatistical analysis ( $5^{\text {th }}$ Edition). Prentice Hall, New Jersey. 472-478 pp.

Zavaleta-Mejía, E. 1999. Management alternatives for plant diseases. Terra Latinoam. 17(3):201-207. 